\title{
Daily temperature fluctuations unpredictably influence developmental rate and morphology at a critical early larval stage in a frog
}

\author{
Juliana M Arrighi ${ }^{1,2}$, Ezra S Lencer ${ }^{1,3}$, Advait Jukar ${ }^{1,4}$, Daesik Park ${ }^{5}$, Patrick C Phillips ${ }^{6}$ and Robert H Kaplan ${ }^{{ }^{*}}$
}

\begin{abstract}
Background: Environmental temperature has profound consequences for early amphibian development and many field and laboratory studies have examined this. Most laboratory studies that have characterized the influence of temperature on development in amphibians have failed to incorporate the realities of diel temperature fluctuations (DTF), which can be considerable for pond-breeding amphibians.

Results: We evaluated the effects of different ecologically relevant ranges of DTF compared with effects of constant temperatures on development of embryos and larvae of the Korean fire-bellied toad (Bombina orientalis). We constructed thermal reaction norms for developmental stage, snout- vent length, and tail length by fitting a Gompertz-Gaussian function to measurements taken from embryos after 66 hours of development in 12 different constant temperature environments between $14^{\circ} \mathrm{C}$ and $36^{\circ} \mathrm{C}$. We used these reaction norms as null models to test the hypothesis that developmental effects of DTF are more than the sum of average constant temperature effects over the distribution of temperatures experienced. We predicted from these models that growth and differentiation would be positively correlated with average temperature at low levels of DTF but not at higher levels of DTF. We tested our prediction in the laboratory by rearing $B$. orientalis embryos at three average temperatures $\left(20^{\circ} \mathrm{C}, 24^{\circ} \mathrm{C}\right.$, and $28^{\circ} \mathrm{C}$ ) and four levels of thermal variation $\left(0^{\circ} \mathrm{C}, 6^{\circ} \mathrm{C}, 13^{\circ} \mathrm{C}\right.$, and $\left.20^{\circ} \mathrm{C}\right)$. Several of the observed responses to DTF were significantly different from both predictions of the model and from responses in constant temperature treatments at the same average temperatures. At an average temperature of $24^{\circ} \mathrm{C}$, only the highest level of DTF affected differentiation and growth rates, but at both cooler and warmer average temperatures, moderate DTF was enough to slow developmental and tail growth rates.

Conclusions: These results demonstrate that both the magnitude of DTF range and thermal averages need to be considered simultaneously when parsing the effects of changing thermal environments on complex developmental responses, particularly when they have potential functional and adaptive significance.
\end{abstract}

Keywords: Bombina orientalis, Amphibian, Diel temperature fluctuation (DTF), Developmental plasticity, Thermal performance curve, Reaction norm

\section{Background}

The relationship between organismal form and function depends on environmental context. For example, developmental rate in ectotherms is contingent on external temperature, and changes in developmental rate can in turn alter the relative allometries of different morphological and physiological traits [1]. The vast majority of

\footnotetext{
* Correspondence: robert.kaplan@reed.edu

'Department of Biology, Reed College, 3203 SE Woodstock Blvd, Portland, OR 97202, USA

Full list of author information is available at the end of the article
}

laboratory studies that infer the effects of environment on development and physiology have been undertaken in constant environments. Yet the thermal environments encountered in nature can be quite variable, and it is unclear whether patterns predicted with models based on observations from constant environments provide an adequate representation of this variation.

A small but growing number of studies have addressed the effects of diel temperature fluctuation (DTF) on amphibian development, which is known to be particularly sensitive to variable environmental inputs [2-6]. The

\section{Biomed Central}


implications of this body of work are multifaceted and include the observation that small increases in average global temperatures may have measurable effects on performance in amphibian embryos and larvae. However, the specific effects of associated daily and seasonal extremes on early amphibian development are less well understood. We contribute to the exploration of the differing developmental responses to environmental variability, focusing on realistic DTF during embryonic development, a period when phenotypic plasticity in response to temperature variation is considered ubiquitous [7-9].

For an embryo developing in a constant temperature environment, a measure of a phenotypic characteristic (e.g., developmental stage or body size) at a given time point can be represented by the functional response of the animal to that temperature, $z(T)$ [10]. When temperature varies, the phenotype will be a complex function of the particular temperature path each individual experiences taken over the age- and/or stage-specific effects of each temperature, $\zeta(\bar{T})$. In other words, temperatures experienced at one time point might affect the individual's response to different temperatures experienced at later time points (i.e., there will be carryover effects). For instance, an individual experiencing a transient temperature exceeding some critical thermal stress limit might experience prolonged period of slowed growth relative to an individual exposed to a lower constant temperature, even if both individuals eventually experience the same average temperature. If variability per se has no effects beyond those caused by the instantaneous effects of temperature experienced at each moment (i.e., there are no carryover effects), then the expected phenotype should simply be a function of the distribution of temperatures experienced, $f(T)$ [10] and the functional response observed at each fixed temperature:

$$
\zeta(\bar{T})=\int f(T) z(T) d T
$$

where the integral is taken over the range of observed temperatures (see also [6,11]). Equation (1) therefore provides a null model against which the hypothesis that temperature variation is more than the sum of individual temperature effects can be tested.

Much of the work on the effects of temperature fluctuations on development has been concerned with predicting developmental rates for ectotherms. Both models and empirical data have shown that temperature fluctuations can increase, decrease, or have no effect on developmental rates [6,12-20]. An emerging conclusion from such studies has been that temperature fluctuations are ecologically important when individuals experience temporary exposure to temperatures at the limits of their physiologically relevant thermal range $[13,16,18,21]$ and that fluctuations at cooler versus warmer temperatures can have different or opposing effects on developmental traits $[12,16]$.

Amphibian development shows a remarkable amount of plasticity throughout both the embryonic and larval periods (e.g., [22,23]). The timing of hatching and larval morphology at hatching can be influenced by a variety of maternal and external-environmental effects [24-26]. Among these environmental effects, temperature is perhaps the most pervasive ([27]; but see [28] and [29] for a discussion of the importance of maternal effects per se). Temperature effects drive adaptation on small geographic scales and short periods of time [30,31] and influence the timing and effectiveness of other environmentally cued hatching mechanisms [32].

At a proximate level, temperature affects the cellular and biochemical rates of differentiation and growth during development [33-35]. At the whole-organism level, environmental temperatures can interact with an individual's genome to affect the amount of time spent developing, body shape, body size, and mass at metamorphosis, as well as trait values for a variety of other phenotypic characters at both the larval and adult stages (e.g. $[23,36])$. For example, in the frog Limnodynastes peronii (Duméril and Bibron, 1841) and the newt Triturus alpestris (Laurenti, 1768), DTF increased developmental rate and was shown to positively affect individual performance relative to developing in a constant temperature environment $[3,4]$. In L. peronii, DTF also increased tadpole body length measurements and decreased metamorph mass, indicating that fluctuating temperature environments can also affect morphology and body size.

Similarly, in the frog Bombina orientalis (Boulenger, 1890), warmer developmental temperatures have been shown to cause tadpoles to hatch at a younger developmental stage $[2,37]$. Moreover, warmer temperatures result in hatchling tadpoles with shorter tail lengths but longer snout-vent lengths [2,38]. Further, embryos experiencing greater amounts of temperature fluctuation in natural breeding ponds tended to hatch at a younger developmental stage and had longer snout-vent length measurements and shorter tail length measurements. These traits have been shown to affect tadpole locomotor performance and fitness at hatching stage, since hatchlings with greater tail length to snout-vent length ratios swim faster and have a higher probability of surviving predation [2,39]. Thus, for $B$. orientalis, a heterogeneous thermal environment can induce variation in traits associated with fitness within a population [2].

We combine two approaches, an empirical modelling approach and a comparative experimental approach. Specifically, we (1) establish thermal reaction norms to constant temperature variation in order to generate null models of developmental response in early larval development in $B$. orientalis, and then (2) use comparative, controlled diel 
temperature variation of different magnitudes to investigate whether observed effects are the result of simple additive DTF or whether particular thermal events have unexpected consequences.

\section{Methods}

Bombina orientalis is widely distributed across the Korean Peninsula and ranges into parts of north-eastern China. Breeding populations of $B$. orientalis utilize a variety of permanent and semi-permanent water bodies as oviposition sites, including rice paddies and granite pools along mountainside streams. These breeding sites can differ remarkably in size, depth, permanence, and shading [37]. As a result, the thermal environment experienced among egg masses within and among populations can be highly variable [36] with respect to both average temperature and diel temperature fluctuation (DTF; [2])

\section{Breeding and egg collections}

During the summer of 2006, adult B. orientalis were collected from a rice paddy outside the village of Duc Doo Won near the city of Chuncheon in the Province of KangWon-do in South Korea and maintained as part of a laboratory colony at Reed College in Portland, Oregon. All adults were housed at a constant $24^{\circ} \mathrm{C}$ and fed crickets ad libitum for at least 10 weeks after a prior breeding [37]. Male and female $B$. orientalis pairs were injected intraperitonealy with 250 IU and 375 IU, respectively, of human chorionic gonadotropin [37] and allowed to oviposit overnight. Fertilized eggs were collected the following morning at blastula stage (Gosner [40] stage 8) and placed in glass fingerbowls filled with $20 \%$ Holtfreter's solution in one of the thermal treatments. Fingerbowls were loosely covered to prevent evaporative cooling and illuminated with an LED light that was set to a $12 \mathrm{~h}$ light $12 \mathrm{~h}$ dark schedule. Multiple sibships were pooled and randomly distributed across treatments.

\section{Design of thermal environments}

We raised $B$. orientalis embryos in a total of 16 different constant and fluctuating thermal environments constructed in programmable incubators (HotPack model 317512 with a Watlow F4 controller). Temperature treatments ranged across those naturally experienced by B. orientalis in the field. Thermal maxima and minima were kept within the limits of $10^{\circ} \mathrm{C}$ and $36^{\circ} \mathrm{C}$, since temperatures outside of that range have been observed to cause increased mortality (see [2]). Within each treatment, two data loggers (Onset Hobo data loggers) per treatment measured the water temperature in two different fingerbowls every 15 minutes. Temperature profiles between bowls were not statistically different.

\section{Development under constant temperatures}

In order to assess the thermal reaction norm of an individual over an environmental gradient, organisms are generally maintained at a range of constant environmental conditions in the laboratory $[6,10]$. Over three occasions, twelve constant temperature environments were employed at temperatures between $14^{\circ} \mathrm{C}$ and $36^{\circ} \mathrm{C}$ at $2^{\circ} \mathrm{C}$ increments (i.e. $14,16, \ldots 34,36^{\circ} \mathrm{C}$ ). Forty embryos were distributed across fingerbowls in each treatment, with 10 embryos per bowl in four large fingerbowls so that 480 embryos were used in this phase of the study. The data from this portion was then used to generate models predicting growth and development under fluctuating temperatures as described in the modelling methods section.

\section{Development under fluctuating temperatures}

In order to explore the effects of daily thermal fluctuation, embryos were allowed to develop under eight environmental treatments at four levels of DTF organized into three groupings by average temperature as shown in Figure 1 (panels A, B, C). This includes constant temperature treatments at each average temperature, which were repeated so that the statistical comparisons (described in the statistical methods section) were made against matched sets of randomized embryos. Twentyfour bowls were placed in each thermal condition, with up to 7 tadpoles in each bowl. In the case of the constant temperature treatment at $24^{\circ} \mathrm{C}$, a controlled temperature room was utilized $\left(24^{\circ} \mathrm{C}\right.$ approx. $+/-0.5^{\circ} \mathrm{C}$; Figure $\left.1 \mathrm{~A}\right)$. The fluctuating temperature treatments at $24^{\circ} \mathrm{C}$ included a low DTF environment $\left(24^{\circ} \mathrm{C}\right.$ approx. $\left.+/-3^{\circ} \mathrm{C}\right)$, a medium DTF environment $\left(24^{\circ} \mathrm{C}\right.$ approx. $\left.+/-6^{\circ} \mathrm{C}\right)$ and a high DTF $\left(24^{\circ} \mathrm{C}\right.$ approx. $\left.+/-10^{\circ} \mathrm{C}\right)$. These three thermally varying environments were established within a single incubator that was programmed to ramp between $14^{\circ} \mathrm{C}$ and $38^{\circ} \mathrm{C}$ every 24 hours with a variable amount of Styrofoam insulation. Fingerbowls in the low temperature variation treatment group (l) were insulated with a Styrofoam cooler, fingerbowls in the medium temperature variation treatment group $(\mathrm{m})$ were insulated with a plastic box and Styrofoam packing material, and fingerbowls in the high temperature variation treatment group $(\mathrm{h})$ were placed in the incubator without any insulation. At average temperatures of each $20^{\circ} \mathrm{C}$ and $28^{\circ} \mathrm{C}$ DTF treatments were created using the same incubator profile, decreased or increased by $4^{\circ} \mathrm{C}$, in separate incubators run synchronously. The fingerbowls in these treatments were insulated in the same way as medium variation treatment from the $24^{\circ} \mathrm{C}$ grouping. DTF treatment profiles for the second two groupings 

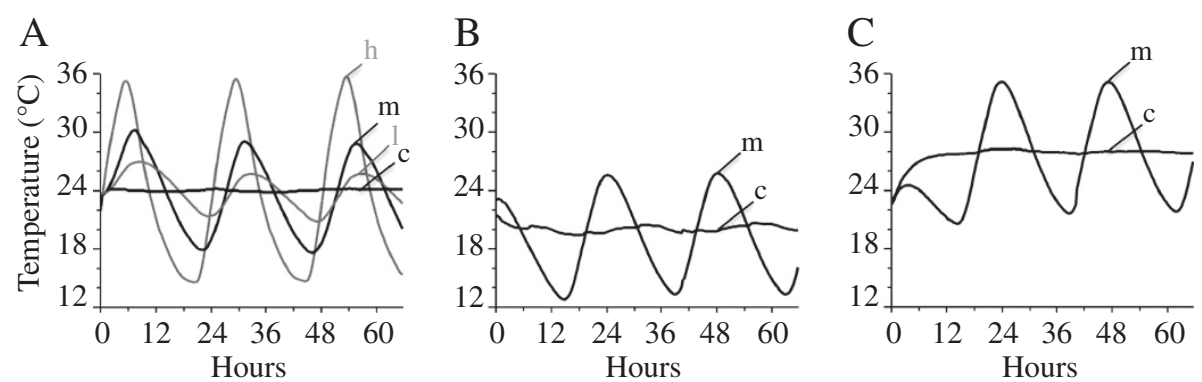

Figure 1 Profiles of fluctuating temperature environments experienced by embryos in this study. Temperature was monitored every 15 minutes and averaged from two data loggers in each thermal environment. Panel $\mathbf{A}$ shows a matched set of four treatments with average temperatures of $24^{\circ} \mathrm{C}$. Panels $\mathbf{B}$ and $\mathbf{C}$ each show a matched set of two treatments with average temperatures of $20^{\circ} \mathrm{C}$ and $28^{\circ} \mathrm{C}$, respectively. Lowercase letters refer to the magnitude of DTF in each treatment: constant temperature $(\mathbf{c})$, low variation (I), medium variation $(\mathbf{m})$, or high variation (h). See text for a more detailed description of these treatments.

are shown in the second two panels (average $20^{\circ} \mathrm{C}$ and $28^{\circ} \mathrm{C}$, respectively; Figure $1 \mathrm{~B}, \mathrm{C}$ ).

\section{Measurement of developmental stage and morphology}

Studies of temperature and development in amphibians often use life history stages as observational markers. We use the developmental stage range where hatching occurs in the field. There are many environmental signals that influence the time and stage at which tadpoles hatch (e.g., $[32,41])$. In field populations, Bombina orientalis can hatch as early as Gosner [40] stage 19, but most hatching occurs during the time when the cornea becomes transparent between Gosner stages 20 and 21 ([39,40]; Figure 2). In laboratory environments $B$. orientalis embryos reach field hatching stages after 66 hours of development at constant $24^{\circ} \mathrm{C}$ but often do not hatch until later stages (personal observation). Because of this observation, embryos in all experiments described here were allowed to develop in all temperature treatments for 66 hours, at which point they were removed from temperature treatments, manually hatched if necessary, photographed and staged under a dissection microscope.

In all cases, for both constant and DTF experiments, at 66 hours of development tadpoles were staged and photographed alongside a $\mathrm{mm}$ ruler using an Olympus digital camera interfaced to a stereomicroscope. Embryos and larvae were staged after Gosner [40], with the following modification. Stages 20 to 21 were further subdivided into 4 stages corresponding to the degree of corneal transparency ([39,42]; Figure 2).

Morphological measurements were then taken from photographs in either Adobe Photoshop or ImageJ [43] after checking for scaling artifacts between the software. We measured total length, defined as the length from the tip of the snout to the end of the tail, snout- vent length, defined as the length from the tip of the snout to a point at the center of the tadpole perpendicular to the posterior margin of the vent, and tail length defined as the length from center of the tadpole perpendicular to the posterior margin of the vent to the tip of the tail, all with respect to the ruler photographed with the tadpole.

\section{Modelling methods}

Mean responses from development under constant temperature experiments were used to estimate the temperature response function for each snout-vent length, tail length, and stage $(z(T)$; Equation 1). Because thermal reaction norms typically fall off beyond critical thermal maxima and minima, we fit each reaction norm using several different nonlinear functions, distinguishing among the models using the Akaike Information Criterion [6,44]. We compared three different functions: quadratic, Gaussian, and Gompertz-Gaussian, respectively specified by

$$
\begin{aligned}
& z T=a T^{2}+b T+c \\
& z T=a \exp \left(-(T-b)^{2} / c\right) \\
& z T=a \exp \left(-e^{b(T-c)-8}-(T-c)^{2} / d\right)
\end{aligned}
$$

Other nonlinear functions suggested by [6] (modified Guassian, Weibull and beta) did not yield convergent solutions for our data. In contrast, the Gompertz-Gaussian suggested by Martin and Huey [11] performed much better and provided an extremely good fit to the data. The predicted effects of thermal variation over these thermal reaction norms (Equation 1) was calculated using the convolution of the estimated thermal reaction norm and the distribution of the thermal variation profiles for each temperature treatment assumed to be uniform for simplification.

All calculations were performed using Mathematica, with the nonlinear fitting and associated statistics being calculated using the NonLinearModelFit function [45]. Errors on the predicted responses were calculated using a bootstrapping approach in which individual temperaturedependent responses were resampled with replacement and then used to recalculate the best-fitting reaction 


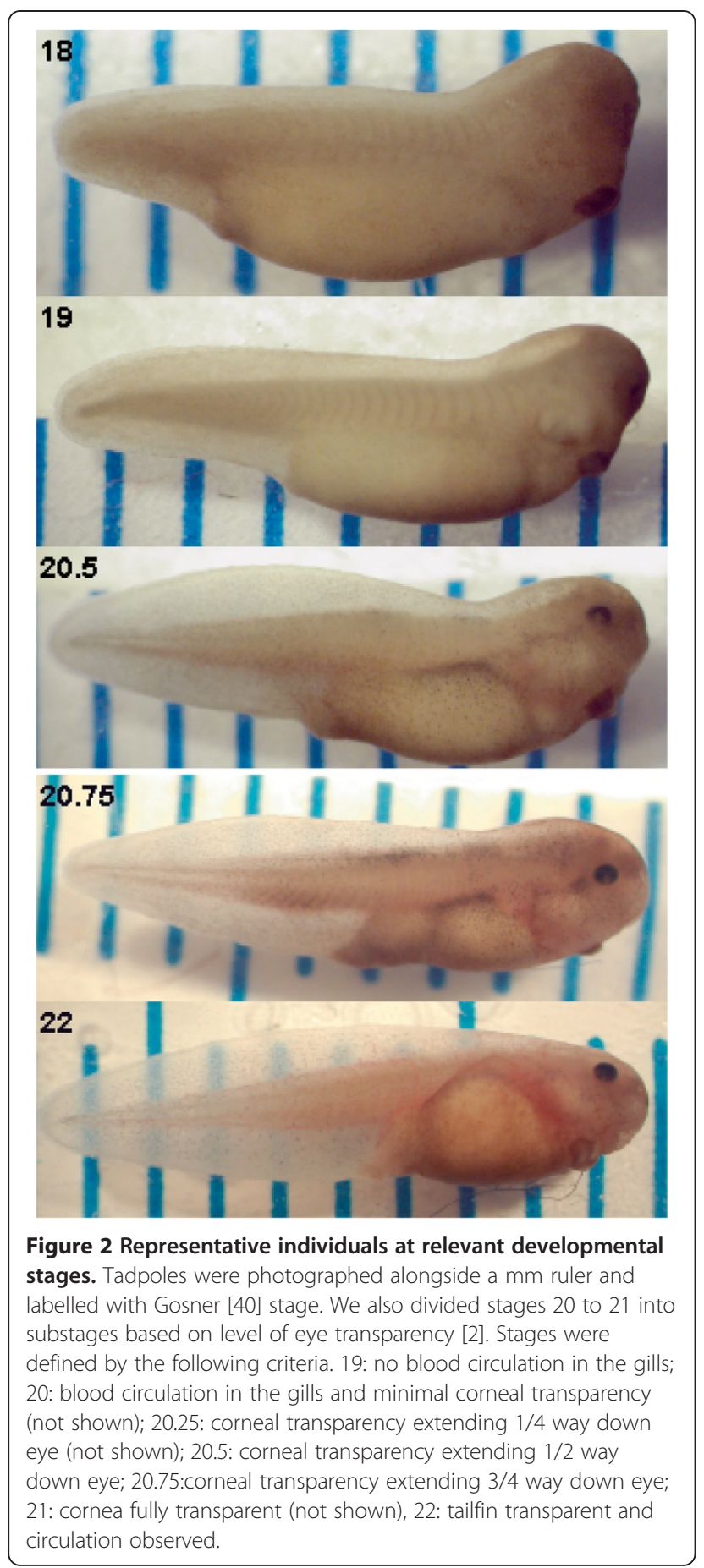

norm. The data were resampled 10,000 times and used to construct an error distribution from which the standard error was calculated.

\section{Statistical methods}

In all cases, fingerbowl averages rather than raw measurements of individual were used to avoid any issues related to pseudo-replication, and we conducted all analyses using the mean value for each fingerbowl. All statistical tests were performed with JMP Version 7 [46]. Data were checked for normality and no transformations were found to be necessary. To test for the effect of DTF we investigated each average temperature separately. We tested whether our DTF treatments were significantly different from their matched constant temperature treatment. Temperature variation was entered as a two or four level fixed effect. In all instances we conducted an ANOVA to test for significant differences among our treatments and a posteriori tests using Tukey-Kramer LSD.

\section{Results}

\section{Modelling effects of DTF based on observations at constant temperatures}

Embryos raised over the range of constant temperatures from 14 to $36^{\circ} \mathrm{C}$ displayed thermal reaction norms for developmental stage, snout-vent length and tail length that are largely typical for ectotherms (Figure 3). Developmental rate was much lower at temperatures below $16^{\circ} \mathrm{C}$, and at $14^{\circ} \mathrm{C}$ embryos only developed to early neurulation (Figure 3A). Since snout-vent length and tail length are defined in terms of an embryo's vent, those measurements cannot be measured until the structure develops, which occurs at early tail bud stage (stage 17) at 66 hours into development at $16^{\circ} \mathrm{C}$. Constant temperatures of $36^{\circ} \mathrm{C}$ were lethal for embryos, which was interpreted as having a value of zero for all measures when fitting the data. Developmental rate after 66 hours, as a function of temperature, increases monotonically until approximately $34^{\circ} \mathrm{C}$ when it plateaus and then abruptly declines. Snoutvent length behaves similarly (Figure 3B), but tail length shows a decline in growth rate beginning at temperatures of $30^{\circ} \mathrm{C}$ (Figure 3C). The reaction norms are well fit by the Gompertz-Gaussian model, but not by Gaussian or quadratic models (coefficients of determination of the fit models exceed 0.99 for all traits; Table 1 ).

The fit reaction norms (Figure 3, A-C) can be used to predict developmental outcomes for each trait over continuously varying levels of DTF for the three different average temperatures and DTF's used in our experiments (Figure 3, D-F). Using this approach, developmental rate is predicted to be relatively insensitive to DTF at the average temperature of $24^{\circ} \mathrm{C}$ until a DTF range of approximately $20^{\circ} \mathrm{C}$ is exceeded (Figure 3D). However, DTF range greater than $13^{\circ} \mathrm{C}$ is expected to influence developmental rate to 66 hours at both cooler $\left(20^{\circ} \mathrm{C}\right)$ and warmer $\left(28^{\circ} \mathrm{C}\right)$ average temperatures. A similar pattern is observed for snout-vent length (Figure 3E) where non-linear effects of DTF are not predicted at an average of $24^{\circ} \mathrm{C}$ until DTF spans of approximately $20^{\circ} \mathrm{C}$ (Figure $3 \mathrm{E}$ ), whereas at average temperatures of $20^{\circ} \mathrm{C}$ and $28^{\circ} \mathrm{C}$ effects emerge at smaller DTF ranges. In contrast, based on the responses to constant temperature environments, increased DTF is predicted to barely change 

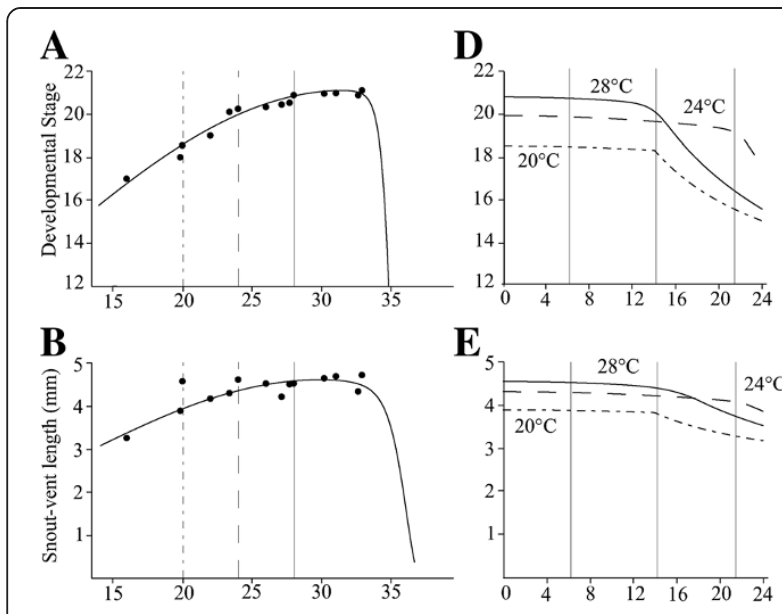

$\mathbf{E}$
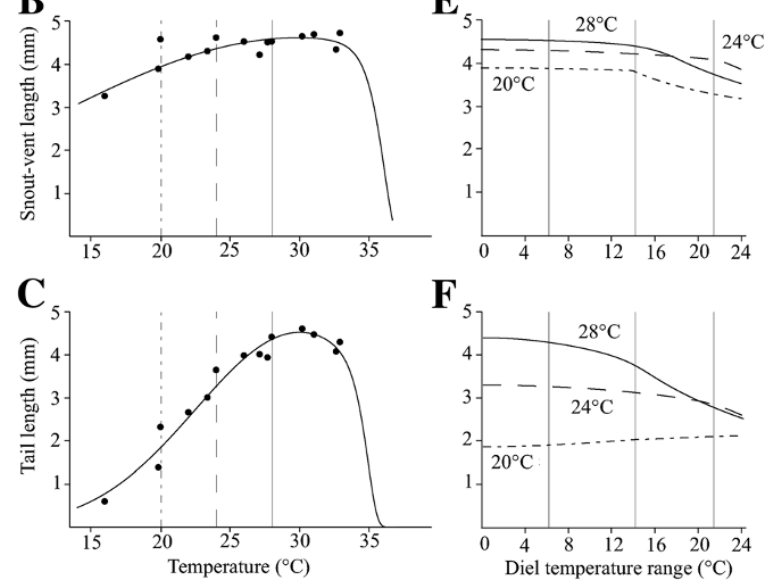

Figure 3 Constant temperature reaction norms and predictions across fluctuating temperature ranges for three average temperatures. Points in panels $\mathbf{A}-\mathbf{C}$ show means for in measured response variables in each constant temperature after 66 hours. In each case, the best fitting model for the reaction norm was a Gompertz-Gaussian function $\left(R^{2}>0.99\right)$, using parameters in Table 1 and described in the text. Vertical lines on panels $\mathbf{A}-\mathbf{C}$ are drawn at average temperatures used in variable temperature treatments (solid: $28^{\circ} \mathrm{C}$ average; dashed: $24^{\circ} \mathrm{C}$ average; dot-dash: $20^{\circ} \mathrm{C}$ average). Panels D-F show the predictions for the variable temperature experiments, assume a uniform distribution of temperatures within the diel variation range and integrating the reaction norms over the experimentally induced environmental variation (Equation 1). Vertical lines in panels $\mathbf{D}$ - $\mathbf{F}$ indicate approximate temperature range locations of the $6^{\circ} \mathrm{C}, 14^{\circ} \mathrm{C}$, and $22^{\circ} \mathrm{C}$ variation treatments in the DTF experiments (I, $\mathbf{m}$, and $\mathbf{h}$ in Figure 1$)$. Error bars show $+/-1$ standard error.

tail growth relative to the average temperature response for constant low temperatures, but to lead to decreased growth as the average constant temperature increases (e.g., $28^{\circ} \mathrm{C}$; Figure 3F).

\section{Description of DTF treatments}

For treatments with average temperatures of $24^{\circ} \mathrm{C}$ the mean temperatures for all four environments were within $0.24^{\circ} \mathrm{C}$ of each other (Figure 1A). The high DTF treatment extended between $9^{\circ} \mathrm{C}$ and $11^{\circ} \mathrm{C}$ above and below the mean. It did not reach $36^{\circ} \mathrm{C}$, where $100 \%$ mortality was observed in the constant temperature treatment. At both the averages of $20^{\circ} \mathrm{C}$ (Figure $1 \mathrm{~B}$ ) and $28^{\circ} \mathrm{C}$ (Figure $1 \mathrm{C}$ ), DTF treatments approximated the medium fluctuation level of the average $24^{\circ} \mathrm{C}$ experiment, extending between $6^{\circ} \mathrm{C}$ and $7^{\circ} \mathrm{C}$ above and below the mean. The shapes of the
Table 1 Model statistics and parameters

\begin{tabular}{cccr}
\hline & Stage & Snout-vent length & Tail length \\
\hline $\begin{array}{l}\text { Quadratic } \\
\text { AIC }\end{array}$ & 1080.7 & 446.6 & 202.3 \\
$\begin{array}{c}\text { Gaussian } \\
\text { AIC }\end{array}$ & 1039.6 & 403.4 & 208.9 \\
Gompertz-Gaussian & & \\
AIC & 298.5 & 224.7 & 93.8 \\
R2 & 0.99 & 0.99 & 0.99 \\
A & 21.13 & 4.55 & 4.62 \\
B & 2.33 & 1.60 & 1.31 \\
C & 31.72 & 30.10 & 30.33 \\
D & 1068.82 & 112.95 & 654.34 \\
\hline
\end{tabular}

Model selection and parameter fitting for the functional reaction norms. In each case, the Gompertz-Gaussian provided the best fit (as indicated by the low AIC value).

three medium fluctuation profiles were similar (" $\mathrm{m}$ " in Figure $1, \mathrm{~A}-\mathrm{C})$. The $20^{\circ} \mathrm{C}$ and $28^{\circ} \mathrm{C}$ constant temperature treatments and their matched, medium DTF treatment groups also had similar average temperatures differing by $1.18^{\circ} \mathrm{C}$ and $0.83^{\circ} \mathrm{C}$, respectively.

\section{Comparative experimental effects of DTF on developmental stage}

When development was terminated after 66 hours, all embryos or hatched larvae were between Gosner [40] stages 18 and 22 (within the range of stages that $B$. orientalis are known to hatch at in the field; [39]; and Figure 2). When tadpoles from constant temperature environments were compared to each other, the embryos from warmer constant temperature environments reached a later developmental stage in the same period of time (i.e., 66 hours; Figure 4A; $F_{2,62}=81.36, P<0.01$ ).

We tested for the effect of variable DTF on developmental stage by investigating each average temperature treatment separately. At $24^{\circ} \mathrm{C}$ increasing DTF had a significant effect on stage $\left(F_{3,87}=11.68, P<0.01\right)$, but only tadpoles that developed in the high variation treatment were found to be at an earlier developmental stage at 66 hours (Figure 4A; Tukey HSD, $P<0.05$ ). In contrast, at both $20^{\circ} \mathrm{C}$ and $28^{\circ} \mathrm{C}$, embryos raised in medium DTF were less differentiated after 66 hours of development than tadpoles from their respective constant temperature treatments (Figure $4 \mathrm{~A} ; 20^{\circ} \mathrm{C}, F_{1,42}=11.23, P<0.01 ; 28^{\circ} \mathrm{C}$ : $\left.F_{1,42}=4.46, P<0.05\right)$.

\section{Comparative experimental effects of DTF on morphology}

In contrast to developmental stage, snout-vent length varied little across different constant temperatures $\left(\mathrm{F}_{2,57}=\right.$ $0.72, \quad P=0.49$; Figure $4 \mathrm{~B})$ whereas, higher constant temperature did lead to increased tail length concordant with constant temperature effects on developmental stage 


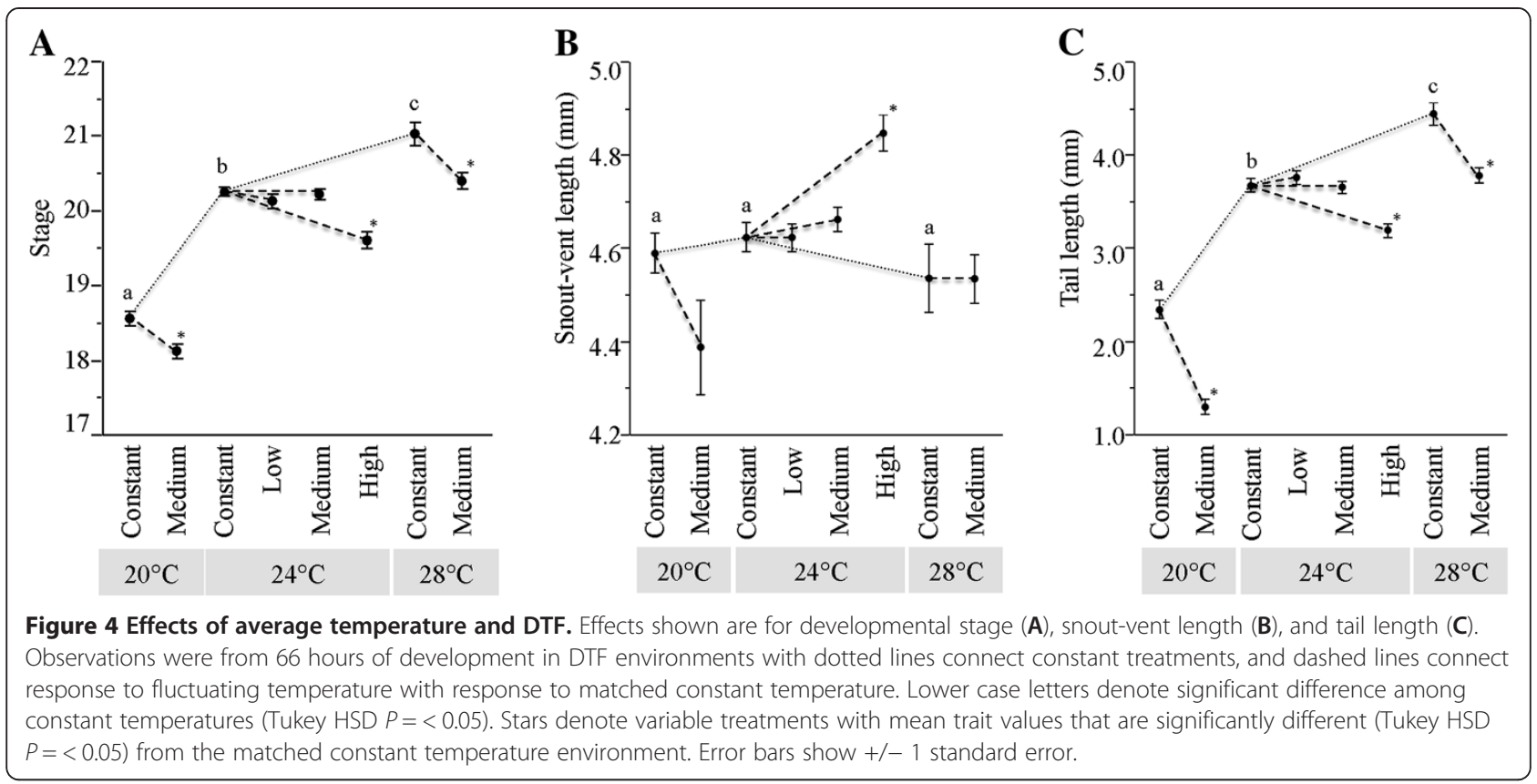

$\left(F_{2}, \quad{ }_{57}=117.6, \quad P<0.01\right.$; Figure $\left.4 \mathrm{C}\right)$. At an average temperature of $20^{\circ} \mathrm{C}$ moderate DTF had an almost significant, negative effect on snout-vent length (Figure 4B; $\left.F_{1,37}=3.38, P=0.07\right)$. Although the mean of the snoutvent lengths of the larvae raised in the DTF treatment was lower, the distribution was wider and encompassed that of the larvae raised at constant $20^{\circ} \mathrm{C}$. There was no significant effect of DTF on snout-vent length at an average temperature of $28^{\circ} \mathrm{C}$ (Figure $4 \mathrm{C} ; F_{1}, 40=0.05, P=0.99$ ). However, at an average temperature of $24^{\circ} \mathrm{C}$, high levels of DTF did significantly increase average snout-vent length $\left(\mathrm{F}_{3,74}=9.91, P<0.01\right.$; Tukey HSD $P<0.01$; Figure $\left.4 \mathrm{~B}\right)$.

DTF had a significant negative effect on tail length at all three average temperatures $\left(20^{\circ} \mathrm{C}: \mathrm{F}_{1,37}=66.98, P<0.01\right.$; $24^{\circ} \mathrm{C}: \mathrm{F}_{3}, 72=9.60, P<0.01 ; 28^{\circ} \mathrm{C}: \mathrm{F}_{1}, 40=21.83, P<0.01$; Figure $4 \mathrm{C}$ ). For the $20^{\circ} \mathrm{C}$ and $28^{\circ} \mathrm{C}$ treatments, the medium DTF environment had significantly shorter tails than their matched constant temperature treatment groups. However, at average $24^{\circ} \mathrm{C}$ only, the high DTF environment was observed to have a significant negative effect on tail length (Tukey HSD $P<0.01$ ).

\section{Comparison of predicted and observed effects of DTF}

The effects of variation in DTF predicted from the models that were generated from thermal reaction norms obtained at constant temperatures are all qualitatively similar to those observed in our DTF experiments for developmental stage at 66 hours (Figure 5A). The lack of concordance is quantitative, with larger than predicted declines at an average temperature of $20^{\circ} \mathrm{C}$ with medium DTF (Tukey HSD $P<0.05)$ and smaller than predicted declines at an average temperature of $28^{\circ} \mathrm{C}$ with medium DTF. For both the predicted and observed cases the largest effects of DTF occur when embryos experience extreme temperatures (Tukey HSD $P<0.05$ ). Low and moderate amounts of DTF at moderate average temperatures have little effect.

In contrast to developmental stage, the predicted effects of variation in DTF on snout-vent length are quite different from those actually observed (Figure 5B). The largest discrepancy is generated by an unexpected increase in snout-vent length at high DTF within the $24^{\circ} \mathrm{C}$ treatment (Tukey HSD $P<0.05$ ), coupled with the lack of sensitivity in the experimental comparisons in the medium and low DTF condition for that same average temperature. In addition, at a low average temperature of $20^{\circ} \mathrm{C}$ there was a greater than predicted decline in snout-vent length as well (Figure 5B).

The discrepancies between observed and predicted effects of DTF on tail length are quite different from snoutvent length but similar to those seen in developmental rate (Figure 5). In particular, tail length showed a greater decline under medium DTF conditions at $20^{\circ} \mathrm{C}$ especially in light of the modelling approach predicting a positive effect. The remainder of comparisons between observed and predicted all showed a decline in tail length with increasing DTF culminating in a similar decline for both at $28^{\circ} \mathrm{C}$ with a medium level of DTF (Tukey HSD $P<0.05$ ).

\section{Discussion}

For many amphibians, timing and condition at the transition from non-motile embryonic development to a freeswimming larval phase at hatching is critical in determining offspring survival $[32,41,47,48]$. Embryonic and larval environments have been shown to influence larval traits such 
as tail length, snout-vent length, body shape, physiological features (e.g., burst speed; [49-51] and predator escape behaviour $[2,38,39,52]$. Thus, it is not surprising that the phenotype of a tadpole at the onset of hatching has been shown to affect its probability of surviving predation in the field [2]. In addition, life-history theory predicts that numerous features of the hatchling tadpole phenotype should be under strong selective control [53] and will probably include carryover effects to later stages of development $[2,5,54]$.

Based on our empirical observations [2] and those of others $[3,4]$ and on recent modelling approaches that stem from computation of developmental outcomes as predicted from thermal reaction norms in a variety of ectotherms [6,10,11,55-57], we predicted that DTF would affect developmental and growth rates in our study. Here we show that temperature treatments whose range extended to the maximum or minimum temperatures of this experiment diminished differentiation and tail growth rates. We also found that models derived from data measured in constant temperature environments did not predict the outcomes of DTF for snout-vent length at high temperatures or differentiation rate and tail length at cold temperatures. The high DTF treatment at average $24^{\circ} \mathrm{C}$ exposed developing larvae to temperatures near their limit of tolerance. Even though the larvae only spent a short time in this region the carryover effects may be substantial. However, the larvae from the moderate DTF treatment at an average of $28^{\circ} \mathrm{C}$, which were also exposed to these extreme temperatures, did not show an increase in snout-vent length. This suggests that amount of DTF, not just temperature experienced, also has an effect on development and growth.

In 2006, Kaplan and Phillips [2] showed in a large field study with $B$. orientalis in Korea that a broad range of naturally occurring daily thermal fluctuations both directly impact the probability of surviving predation and interact with maternal effects (i.e., ovum size; see also [26]) in non-additive ways. In that same year Niehaus, Wilson and Franklin [3] compared measures of growth and performance in the laboratory between one level of DTF and one corresponding constant temperature in the Australian marsh frog, Limnodynastes peronii and found increased growth throughout development, reduced development time to metamorphosis, and increased post-metamorphic jumping ability, for those embryos and larvae reared in the thermally fluctuating environment. Thus, there is evidence that naturally occurring DTF is important in many aspects of amphibian development. Later studies [5] found that early exposure to DTF did not tend to change sensitivity (i.e., carryover) of physiological function later in development but punctuated the important need to come to understand the role of fluctuating thermal environments "under naturalistic thermal conditions" when considering thermal adaptation and evolution [6]. In addition, Meřaková \& Gvoždík [4] also recently explored three levels of DTF at one average temperature in the European newt, Triturus alpestris. They found that higher DTF during embryogenesis increased swimming speed at the hatching stage relative to 
development at lower DTF, but only during a performance test at a cooler temperature. Thus, prior results (and those reported here) show important effects of development in a DTF environment that are dependent on the particular temperatures experienced, the range of temperatures experienced, the developmental stages at which they are experienced, and species.

Studies in insects and reptiles that have relied on empirical modelling approaches have also found that the effects of DTF depend on the magnitude of this variation combined with variation in average temperature (insects: $[12,18]$; reptiles: $[13,15,16,21,58-63]$. In many cases the relationship between temperature and developmental rate (or growth rate) becomes non-linear near the limits of a species thermal tolerance range, and models predict that temperature fluctuations should become ecologically important and less predictable when individuals experience temporary exposure to temperatures near these limits for the species (e.g. $[18,64])$.

In the case of our study, three conditions, our $20^{\circ} \mathrm{C}$ medium DTF, $24^{\circ} \mathrm{C}$ high DTF and $28^{\circ} \mathrm{C}$ medium DTF treatments (Figure 1) transiently expose developing embryos to extreme temperatures where physiological functions that regulate cellular and organismal homeostasis may become impeded. These extreme temperature fluctuations can uncouple differentiation rates and growth rates and further uncouple growth rates for different body regions resulting in body allometry effects. This offers an important explanation for our prior results of increased egg size (a maternal effect and constrained to impacting snout-vent length primarily) decreasing offspring performance via sprint speed under more extreme thermal conditions so that tail to snout-vent length ratios go down dramatically under extreme transient heat exposure in embryos $[2,38,39]$.

\section{Conclusions}

In this study, the biggest difference between predictions based on data from constant temperature environments and observations from development in fluctuating environments was largest when physiological thermal limits were approached. Overall, we find that the difference in levels of temperature variation is important both because of variability per se and because of the physiological temperature limits that alter the trajectory of early development in this frog in ways not predicted by our null model.

Recent anthropogenically caused environmental change, including global changes in temperature regimes, offer numerous opportunities for basic research and challenges for conservation. For example, across its range on the Korean peninsula, $B$. orientalis utilizes a variety of oviposition sites that represent a heterogeneous thermal landscape with respect to both average temperature and DTF. In addition to the yet unclear relationships between thermal fluctuations and global warming, recent diminishment and changes in rice agricultural practices have the potential to dramatically shift the thermal landscape with respect to DTF $([65,66]$ respectively). Prior field results [2] together with the laboratory studies reported here, show that varying levels of DTF in relation to average temperatures can dramatically affect fitness related traits for $B$. orientalis.

Changing thermal landscapes and their impacts on organism adaptability and concomitant distribution, abundance, and evolution also offer unique opportunities to study the dynamics of local adaptation [67] and the rapidity and intensity of natural selection in promoting adaptation $[68,69]$. Because ectotherms are particularly vulnerable to the developmental ramifications of temperature changes, studies of amphibian embryos and larvae and their development in fluctuating thermal environments can provide important insights towards these goals (see [70]). Amphibians can play an important role in advancing both the theoretical understanding of the development and evolution of thermal adaptation but perhaps more critically in advancing our understanding of amphibian conservation in changing thermal landscapes [6,52,71].

\section{Abbreviations}

DTF: Diel temperature fluctuation.

\section{Competing interests}

The authors declare that they have no competing interests.

\section{Authors' contributions}

JMA, ESL, and AJ carried out the experimental work. PCP carried out the modelling work. JMA, ESL, AJ, PCP, and RHK analyzed the data. DP provided logistical support with $B$. orientalis. All authors wrote, read and approved the final manuscript.

\section{Acknowledgements}

We are grateful to various members of the Kaplan and Park Labs for their help in animal husbandry, breeding, and data collection, including Linsey Arnold, Kristy Gonyer, Martha Baugh-Vonreis, Morgan Movius and Jessie Ellington at Reed College. Ja-Kyung Kim, Jung- Hyun Lee, and Nam-Yong Ra at Kangwon National University in Chuncheon, Korea. The manuscript was improved with the assistance of Linda Doyle and several anonymous referees and with further logistical support from Miriam Seger at Think Tank, Joshua Tree, CA. Frogs were imported under a permit from the USFWS and housed at Reed College under permit from the ODFW. Experiments followed protocol approved by Reed College Institutional Animal Care Committee. Research was supported by grants from Howard Hughes Medical Institute, James F. and Marion L. Miller Foundation, Andrew W. Mellon Foundation and Reed College Faculty Development Research Fund.

\section{Author details}

${ }^{1}$ Department of Biology, Reed College, 3203 SE Woodstock Blvd, Portland, OR 97202, USA. ${ }^{2}$ Systems Science, Portland State University, Portland, OR, USA. ${ }^{3}$ Ecology and Evolutionary Biology, Cornell University, Ithaca, NY, USA. ${ }^{4}$ Environmental Science and Policy, George Mason University, Fairfax, VA, USA. ${ }^{5}$ Division of Science Education, Kangwon National University, Chuncheon, Kangwon 200-701, South Korea. ${ }^{6}$ Institute of Ecology and Evolution, University of Oregon, Eugene, OR 97403, USA. 


\section{References}

1. Spicer Jl, Burggren WW: Development of physiological regulatory systems: altering the timing of crucial events. Zoology 2003, 106:91-99.

2. Kaplan RH, Phillips PC: Ecological and developmental context of natural selection: maternal effects and thermally induced plasticity in the frog Bombina orientalis. Evolution 2006, 60:142-156.

3. Niehaus AC, Wilson RS, Franklin CE: Short- and long-term consequences of thermal variation in the larval environment of anurans. J Anim Ecol 2006, 75:686-692.

4. Meřaková E, Gvoždík L: Thermal acclimation of swimming performance in newt larvae: the influence of diel temperature fluctuations during embryogenesis. Funct Ecol 2009, 23:989-995.

5. Niehaus AC, Wilson RS, Seebacher F, Franklin CE: Striped marsh frog (Limnodynastes peronii) tadpoles do not acclimate metabolic performance to thermal variability. J Exp Biol 2011, 214:1965-1970.

6. Niehaus AC, Angilletta MJ Jr, Sears MW, Franklin CE, Wilson RS: Predicting the physiological performance of ectotherms in fluctuating thermal environments. J Exp Biol 2012, 215:694-701.

7. Gilchrist GW: Specialists and generalists in changing environments. I. Fitness landscapes of thermal sensitivity. Am Nat 1995, 146:252-270.

8. Travis J, McManus MG, Baer CF: Sources of variation in physiological phenotypes and their evolutionary significance. Am Zool 1999, 39:422-433.

9. Angilletta MJ: Thermal adaptation: a theoretical and empirical synthesis. New York: Oxford University Press; 2009.

10. Kingsolver JG, Gomulkiewicz R: Environmental variation and selection on performance curves. Integr Comp Biol 2003, 43:470-477.

11. Martin PR, Huey RB: Why "suboptimal" is optimal: Jensen's inequality and ectotherm thermal preferences. Am Nat 2008, 171:E102-E118.

12. Hagstrum DW, Milliken GA: Modeling differences in insect developmental times between constant and fluctuating temperatures. Annals Entomol Soc Am 1991, 84:369-379.

13. Shine $R$, Harlow PS: Maternal manipulation of offspring phenotypes via nest-site selection in an oviparous lizard. Ecology 1996, 77:1808-1817.

14. Webb JK, Brown GP, Shine R: Body size, locomotor speed and antipredator behaviour in a tropical snake (Tropidonophis mairii, Colubridae): the influence of incubation environments and genetic factors. Funct Ecol 2001, 15:561-568.

15. Mullins MA, Janzen FJ: Phenotypic effects of thermal means and variances on smooth softshell turtle (Apalone mutica) embryos and hatchlings. Herpetologica 2006, 62:27-36.

16. Du W-G, Feng J-H: Phenotypic effects of thermal mean and fluctuations on embryonic development and hatchling traits in a lacertid lizard, Takydromus septentrionalis. J Exper Zool Part a-Ecol Genet Physiol 2008, 309A:138-146.

17. Ragland GJ, Kingsolver JG: The effect of fluctuating temperatures on ectotherm life-history traits: comparisons among geographic populations of Wyeomyia smithii. Evol Ecol Res 2008, 10:29-44.

18. Kingsolver JG, Ragland GJ, Diamond SE: Evolution in a constant environment: thermal fluctuations and thermal sensitivity of laboratory and field populations of manduca sexta. Evolution 2009, 63:537-541.

19. Bozinovic F, Bastias DA, Boher F, Clavijo-Baquet S, Estay SA, Angilletta MJJ: The mean and variance of environmental temperature interact to determine physiological tolerance and fitness. Physiol Biochem Zool 2011, 84:543-552.

20. Kingsolver JG: Feeding, growth, and the thermal environment of cabbage white caterpillars, pieris rapae L. Physiol Biochem Zool 2000, 73:621-628.

21. Georges A, Beggs K, Young JE, Doody JS: Modelling development of reptile embryos under fluctuating temperature regimes. Physiol Biochem Zool 2005, 78:18-30.

22. Moore JA: Temperature tolerance and areas of development in the eggs of Amphibia. Ecology 1939, 20:459-478.

23. Alvarez D, Nicieza AG: Effects of temperature and food quality on anuran larval growth and metamorphosis. Funct Ecol 2002, 16:640-648.

24. Bradford DF: Incubation-time and rate of embryonic-development in amphibians - the influence of ovum size, temperature, and reproductive mode. Physiol Zool 1990, 63:1157-1180.

25. Ficetola GF, De Bernardi F: Offspring size and survival in the frog Rana latastei: from among-population to within-clutch variation. Biol J Linn Soc 2009, 97:845-853.

26. Dziminski MA, Vercoe PE, Roberts JD: Variable offspring provisioning and fitness: a direct test in the field. Funct Ecol 2009, 23:164-171.
27. Laugen AT, Laurila A, Merila J: Latitudinal and temperature-dependent variation in embryonic development and growth in Rana temporaria. Oecologia 2003, 135:548-554

28. Pfennig DW, Martin RA: A maternal effect mediates rapid population divergence and character displacement in spadefoot toads. Evolution 2009, 63:898-909.

29. Martin RA, Pfennig DW: Maternal investment influences expression of resource polymorphism in amphibians: implications for the evolution of novel resource-Use phenotypes. PLOS One 2010, 5:1-5.

30. Skelly DK: Microgeographic countergradient variation in the wood frog, Rana sylvatica. Evolution 2004, 58:160-165.

31. Phillimore AB, Hadfield JD, Jones OR, Smithers RJ: Differences in spawning date between populations of common frog reveal local adaptation. Proc Natl Acad Sci U S A 2010, 107:8292-8297.

32. Warkentin KM: Plasticity of hatching in amphibians: evolution, trade-offs, cues and mechanisms. Integr Comp Biol 2011, 51:111-117.

33. Smith-Gill SJ, Berven KA: Predicting amphibian metamorphosis. Am Nat 1979, 113:563-585

34. van der Have TM, DeJong G: Adult size in ectotherms: temperature effects on growth and differentiation. J Theor Biol 1996, 183:329-340.

35. van der Have TM: A proximate model for thermal tolerance in ectotherms. Oikos 2002, 98:141-155.

36. Kaplan $\mathrm{RH}$ : Ovum size plasticity and maternal effects on the early development of the Frog, Bombina-Orientalis Boulenger, in a field population in Korea. Funct Ecol 1989, 3:597-604.

37. Kaplan RH: Developmental plasticity and maternal effects of reproductive characteristics in the Frog, Bombina orientalis. Oecologia 1987, 71:273-279.

38. Kaplan RH: Greater maternal investment can decrease offspring survival in the frog Bombina orientalis. Ecology 1992, 73:280-288.

39. Parichy DM, Kaplan RH: Maternal investment and developmental plasticity - functional consequences for locomotor performance of hatchling Frog larvae. Funct Ecol 1995, 9:606-617.

40. Gosner KL: A simplified table for staging anuran embryos and larvae with notes on identification. Herpetologica 1960, 16:183-190.

41. Warkentin KM: Adaptive plasticity in hatching age - a response to predation risk trade-offs. Proc Natl Acad Sci U S A 1995, 92:3507-3510

42. Glucksmann A: Development and differentiation of the tadpole eye Brit Jour Ophthalmol 1940, 24:153-178.

43. Rasband WS: ImageJ. Bethesda, Maryland, USA: U. S. National Institutes of Health. 2009.

44. Angilletta MJ: Estimating and comparing thermal performance curves. J Therm Biol 2006, 31:541-545.

45. Wolfram Research Inc: Mathematica, version 6.0. Illinois: Champaign; 2007.

46. SAS Institute Inc: JMP, version 7. Cary, North Carolina: SAS Institute, Inc. 2007.

47. Calef GW: Natural Mortality of Tadpoles in a Population of Rana-Aurora. Ecology 1973, 54:741-758.

48. Travis J: Control of larval growth variation in a population of pseudacristriseriata (Anura, Hylidae). Evolution 1981, 35:423-432.

49. Watkins TB, Vraspir J: Both incubation temperature and posthatching temperature affect swimming performance and morphology of wood frog tadpoles (Rana sylvatica). Physiol Biochem Zool 2006, 79:140-149.

50. Johnson JB, Burt DB, DeWitt TJ: Form, function, and fitness: pathways to survival. Evolution 2008, 62:1243-1251.

51. Arendt J: Morphological correlates of sprint swimming speed in five species of spadefoot toad tadpoles: comparison of morphometric methods. J Morphol 2010, 271:1044-1052.

52. Touchon JC, Warkentin KM: Thermally contingent plasticity: temperature alters expression of predator-induced colour and morphology in a Neotropical treefrog tadpole. J Anim Ecol 2011, 80:79-88.

53. Hopkins GR, Gall BG, French SS, Brodie ED: Interfamily variation in amphibian early life-history traits: raw material for natural selection? Ecol Evol 2012, 2:1637-1643.

54. Touchon JC, Warkentin KM: Short- and long-term effects of the abiotic egg environment on viability, development and vulnerability to predators of a Neotropical anuran. Funct Ecol 2010, 24:566-575.

55. Huey RB, Kingsolver JG: Evolution of thermal sensitivity of ectotherm performance. Trends Ecol Evol 1989, 4:131-135.

56. Angilletta MJ, Huey RB, Frazier MR: Thermodynamic effects on organismal performance: is hotter better? Physiol Biochem Zool 2010, 83:197-206. 
57. Angilletta MJ, Sears MW: Coordinating theoretical and empirical efforts to understand the linkages between organisms and environments. Integr Comp Biol 2011, 51:653-661.

58. Shine R, Elphick MJ, Harlow PS: The influence of natural incubation environments on the phenotypic traits of hatchling lizards. Ecology 1997, 78:2559-2568.

59. Ashmore GM, Janzen FJ: Phenotypic variation in smooth softshell turtles (Apalone mutica) from eggs incubated in constant versus fluctuating tempertures. Oecologia 2003, 134:182-188.

60. Niewiarowski PH, Angilletta MJ: Countergradient variation in embryonic growth and development: do embryonic and juvenile performances trade off? Funct Ecol 2008, 22:895-901.

61. Les HL, Paitz RT, Bowden RM: Living at extremes: development at the edges of viable temperature under constant and fluctuating conditions. Physiol Biochem Zool 2009, 82:105-112.

62. Andrewartha SJ, Mitchell NJ, Frappell PB: Does incubation temperature fluctuation influence hatchling phenotypes in reptiles? A test using parthenogenetic geckos. Physiol Biochem Zool 2010, 83:597-607.

63. Oufiero CE, Angilletta MJ: Energetics of lizard embryos at fluctuating temperatures. Physiol Biochem Zool 2010, 83:869-876.

64. Worner SP: Performance of phenological models under variable temperature regimes: consequences of the Kaufmann or rate summation effect. Environ Entomol 1992, 21:689-699.

65. Goto S, Kim HC, Uchida Y, Okubo Y: Reconstruction of the ground surface temperature history from the borehole temperature data in the southeastern part of the Republic of Korea. J Geophys Eng 2005, 2:312-319.

66. Choi K: Vegetation and climate history of the lowland on the Korean peninsula. J Korean Phys Soc 2001, 39:762-765.

67. Kawecki TJ, Ebert D: Conceptual issues in local adaptation. Ecol Lett 2004, 7:1225-1241.

68. Reznick DN, Ghalambor CK: The population ecology of contemporary adaptations: what empirical studies reveal about the conditions that promote adaptive evolution. Genetica 2001, 112:183-198.

69. Hendry AP, Farrugia TJ, Kinnison MT: Human influences on rates of phenotypic change in wild animal populations. Mol Ecol 2008, 17:20-29.

70. Irschick DJ, Meyers JJ, Husak JF, Le Galliard J: How does selection operate on whole-organism functional performance capacities? A review and synthesis. Evol Ecol Res 2008, 10:177-196.

71. Collins JP, Crump ML: Extinction in our times. New York: Oxford University Press; 2009.

doi:10.1186/1472-6785-13-18

Cite this article as: Arrighi et al:: Daily temperature fluctuations unpredictably influence developmental rate and morphology at a critical early larval stage in a frog. BMC Ecology 2013 13:18.

\section{Submit your next manuscript to BioMed Central and take full advantage of:}

- Convenient online submission

- Thorough peer review

- No space constraints or color figure charges

- Immediate publication on acceptance

- Inclusion in PubMed, CAS, Scopus and Google Scholar

- Research which is freely available for redistribution 